\title{
N. DECISION DE 21 DE ABRIL DE 1970 RELATIVA A LA SUSTITUCION DE LAS CONTRIBUCIONES FINANCIERAS DE LOS ESTADOS MIEMBROS POR RECURSOS PROPIOS DE LAS COMUNIDADES
}

\section{EL CONSEJO DE LAS COMUNIDADES EUROPEAS,}

VISTO el Tratado constitutivo de la Comunidad Económica Europea y, en particular, su artículo 201,

VISTO el Tratado constitutivo de la Comunidad Europea de la Energía Atómica y, en particular, su artículo 173 ,

VISTA la propuesta de la Comisión,

VISTO el dictamen de la Asamblea,

VISTO el dictamen del Comité Económico y Social,

CONSIDERANDO que la sustitución total de las contribuciones financieras de los Estados miembros por recursos propios de las Comunidades sólo podrá realizarse en forma progresiva;

CONSIDERANDO que el apartado 1 del artículo 2 del Reglamento número 25 , relativo a la financiación de la polí- tica agrícola común establece que, en la fase de mercado único, se asignen a la Comunidad y se destinen a los gastos comunitarios los ingresos procedentes de las exacciones reguladoras agrícolas;

CONSIDERANDO que el articulo 201 del Tratado constitutivo de la Comunidad Económica Europea menciona expresamente, entre los recursos propios que podrán sustituir a las contribuciones financieras de los Estados miembros, los ingresos procedentes del aran cel aduanero común, tras el establecimiento definitivo de éste;

CONSIDERANDO que conviene atenuar los efectos, en los presupuestos de los Estados miembros, de la transferencia a las Comunidades de los ingresos procedentes de los derechos de aduana; que conviene prever un régimen' que permita llegar progresivamente y en un 
N plazo determinado a la transferencia total;

CONSIDERANDO que los ingresos procedentes de las exacciones reguladoras agrícolas y de los derechos de aduana no son suficientes para garantizar el equilibrio del presupuesto de las Comunidades; que conviene, pues; asignar, además, a las Comunidades ingresos fiscales, siendo los más adecuados los que proceden de la aplicación de un tipo único a la base imponible del impuesto sobre el valor añadido, determinada de manera uniforme para los Estados miembros,

HA ESTABLECIDO LAS PRESENTES DISPOSICIONES, CUYA ADOPCION RECOMIENDA A LOS ESTADOS MIEMBROS:

Art. 1. Se asignarán a las Comunidades recursos propios a fin de garantizar el equilibrio de su presupuesto, con arreglo a las modalidades establecidas en los artículos siguientes.

Art. 2. A partir del 1 de enero de 1971, los ingresos procedentes:

a) De las exacciones reguladoras, primas, montantes suplementarios o compensatorios, importes o elementos adicionales y de los otros derechos que hayan fijado o que vayan a fijar las instituciones de las Comunidades respecto de los intercambios con los paises no miembros, en el marco de la política agrícola común, así como de las cotizaciones y otros derechos previstos en el marco de la organización común de mercados en el sector del azúcar, denominados en lo sucesivo «exacciones reguladoras agricolas»;

b) De los derechos del arancel adua. nero común y de los otros derechos que hayan fijado o que vayan a fijar las instituciones de las Comunidades respecto de los intercambios con los daíses no miembros, denominados en lo sucesivo "derechos de aduana»,

constituirán, en las condiciones previstas en el artículo 3, recursos propios, que se consignarán en el presupuesto de las Comunidades.
Constituirán, asimismo, recursos propios consignados en el presupuesto de las Comunidades, los ingresos procedentes de otros tributos que se establezcan, en el marco de una política común, de conformidad con las disposiciones del Tratado constitutivo de la Comunidad Económica Europea o del Tratado constitutivo de la Comunidad Europea de la Energía Atómica, siempre que se haya aplicado hasta el final el procedimiento del artículo 201 del Tratado constitutivo de la Comunidad Económica Europea o del artículo 173 del Tratado constitutivo de la Comunidad Europea de la Energía Atómica.

Art. 3. 1. A partir del 1 de enero de 1971, los ingresos procedentes de las exacciones reguladoras agrícolas se consignarán en su totalidad en el presupuesto de las Comunidades.

A partir de la misma fecha, los ingresos procedentes de los derechos de aduana se consignarán progresivamente en el presupuesto de las Comunidades.

El importe de los derechos de aduana asignado anualmente a las Comunidades por cada Estado miembro será igual a la diferencia entre un importe de referencia y el importe de las exacciones reguladoras agrícolas asignadas a las Comunidades con arreglo al párrafo primero. En caso de que esta diferencia sea negativa, no habrá lugar al pago de derechos de aduana por parte del Estado miembro de que se trate ni a la devolución de las exacciones reguladoras agrícolas por parte de las Comunidades.

El importe de referencia previsto en el párrafo tercero será igual:

- En 1971 al 50 por 100 ;

- En 1972 al 62,50 por 100 ;

- En 1973 al 75 por 100 ;

- En 1974 al 87,50 por 100 .

- A partir del 1 de enero de 1975 al 100 por 100 ,

del importe total de las exacciones reguladoras agrícolas y de los derechos de aduana percibidos por cada Estado miembro. 
Las Comunidades reembolsarán a cada Estado miembro el 10 por 100 de los importes abonados de conformidad con los párrafos precedentes en concepto de gastos de recaudación.

2. Durante el periodo comprendido entre el 1 de enero de 1971 y el 31 de diciembre de 1974, las contribuciones financieras de los Estados miembros necesarias para garantizar el equilibrio del presupuesto de las Comunidades se determinarán con arreglo a la clave de reparto siguiente:

Bélgica: 6,8.

Alemania: 32,9 .

Francia: 32,6.

Italia: 20,2 .

Luxemburgo: 0,2.

Paises Bajos: 7,3.

3. Sin embargo, durante este mismo periodo, la variación de un año a otro de la parte correspondiente a cada Estado miembro en la totalidad de los importes abonados con arreglo a los apartados 1 y 2 no podrá exceder del 1 por 100 en sentido ascendente ni del 1,5 por 100 en sentido descendente, siempre que estos importes sean tomados en consideración en el marco del párrafo segundo. Para el año 1971, se tomarán como referencia, para la aplicación de esta norma, las contribuciones financieras de cada Estado miembro al conjunto de los presupuestos de 1970 , en la medida en que dichos presupuestos sean tomados en consideración en el marco del párrafo segundo.

Para la aplicación del párrafo primero, se tomarán en consideración, para cada ejercicio, los elementos siguientes:

a) Los gastos relativos a los créditos de pagos acordados para el ejercicio de que se trate, en el marco del presupuesto de investigación y de inversiones de la Comunidad Europea de la Energía Atómica, con exclusión de los gastos relativos a los programas complementarios;

b) Los gastos relativos a los créditos del Fondo Social Europeo;

c) En cuanto al Fondo Europeo de Orientación y de Garantía Agrícola, los gastos relativos a los créditos de la Sección Garantia y los de la Sección Orientación, excepto los créditos consignados o reconsignados para períodos contables anteriores al ejercicio considerado.

Para el año de referencia de 1970 , dichos gastos serán:

- Para la Sección Garantía, los mencionados en el artículo 8 del Reglamento (CEE) número $728 / 70$, del Consejo, de 21 de abril de 1970 , relativo a las disposiciones complementarias para la financiación de la política agricola común.

- Para la Sección Orientación, un importe de 285 millones de unidades de cuenta distribuido con arreglo a la clave de reparto prevista en el artículo 7 de dicho Reglamento.

quedando entendido que, para el cálculo de la parte relativa a Alemania, se tomará como clave de referencia un porcentaje del 31,5 por 100 ;

d) Los restantes gastos relativos a los créditos consignados en el presupuesto de las Comunidades.

Si la aplicación de las disposiciones del presente apartado a uno o a varios Estados miembros ocasionare un déficit en el presupuesto de las Comunidades, el importe de dicho déficit se repartirá para el año considerado entre los demás Estados miembros, dentro de los límites de variación establecidos en el párrafo primero de este apartado y según la clave de reparto de las contribuciones establecida en el apartado 2 . La operación se repetirá, si fuere necesario.

4. La financiación mediante recursos propios de las Comunidades de los gastos relativos a los programas de investigación de la Comunidad Europea de la Energía Atómica no excluirá la consignación en el presupuesto de las Comunidades de los gastos relativos a los programas complementarios, ni la financiación de dichos gastos por medio de contribuciones financieras de los Estados miembros, determinadas según una clave de reparto especial establecida 
en virtud de una decisión del Consejo, tomada por unanimidad.

5. No obstante lo dispuesto en el presente artículo, los créditos consignados en un presupuesto anterior al ejercicio de 1971 y prorrogados hasta un presupuesto posterior o reconsignados en este último serán financiados por medio de contribuciones financieras de los Estados miembros, según las claves de reparto aplicables en el momento de su primera consignación.

Los créditos de la Sección Orientación que, estando consignados por primera vez en el presupuesto de 1971, se refieran a periodos contables del Fondo Europeo de Orientación y de Garantía Agrícola anteriores al 1 de enero de 1971, estarán sujetos a la clave de reparto correspondiente a dichos periodos.

Art. 4. 1. A partir del 1 de enero de 1975, el presupuesto de las Comunidades será, sin perjuicio de otros ingresos, integramente financiado con recursos propios de las Comunidades.

Estos recursos comprenderán los ingresos mencionados en el artículo 2, así como los procedentes del impuesto sobre el valor añadido y obtenidos mediante la aplicación de un tipo que no podrá sobrepasar el 1 por 100 a una base imponible determinada de manera uniforme para los Estados miembros, con arreglo a normas comunitarias. Dicho tipo se fijará en el marco del procedimiento presupuestario. Sin embargo, si al iniciarse un ejercicio no se hubiere aprobado aún el presupuesto, el tipo fijado anteriormente seguirá siendo aplicable hasta la entrada en vigor de otro nuevo.

No obstante, durante el periodo comprendido entre el 1 de enero de 1975 y el 31 de diciembre de 1977, la variación de un año a otro de la parte correspondiente a cada Estado miembro en relación con el año anterior no podrá exceder del 2 por 100 . En caso de que se sobrepasare este porcentaje, se efectuarán las adaptaciones necesarias, dentro de este límite de variación, mediante compensaciones financieras entre los Estados miembros interesados, en forma proporcional a la cuota de ingresos procedentes del impuesto sobre el valor añadido o de las contribuciones financieras mencionadas en los apartados 2 y 3, que cada uno de ellos debe aportar.

2. No obstante lo dispuesto en el párrafo segundo del apartado 1 , si, el 1 de enero de 1975, las normas que determinan la base imponible uniforme del impuesto sobre el valor añadido no fueren todavía aplicadas en todos los Estados miembros, sino únicamente en tres de ellos como mínimo, la contribución financiera al presupuesto de las Comunidades por parte de cada Estado miembro que no aplique aún la base imponible uniforme del impuesto sobre el valor añadido se determinará en función de la cuota de su producto nacional bruto con respecto a la suma de los productos nacionales brutos de los Estados miembros; el saldo del presupuesto será cubierto mediante ingresos procedentes del impuesto sobre el valor añadido conforme al párrafo segundo del apartado 1, percibidos por los demás Estados miembros. Esta excepción dejará de surtir efecto tan pronto como se cumplan las condiciones establecidas en el apartado 1 .

3. No obstante lo dispuesto en el párrafo segundo del apartado 1 , si, el 1 de enero de 1975, las normas que determinan la base imponible uniforme del impuesto sobre el valor añadido no fueren todavia aplicadas en tres Estados miembros como minimo, la contribución financiera al presupuesto de las Comunidades por parte de cada Estado miembro se determinará en función de la cuota de su producto nacional bruto con respecto a la suma de los productos nacionales brutos de los Estados miembros. Esta excepción dejará de surtir efecto tan pronto como se cumplan las condiciones establecidas en el apartado 1 ó 2.

4. A efectos de aplicación de los apartados 2 y 3 , se entenderá por pro- 
ducto nacional bruto, el producto nacional bruto al precio de mercado.

5. A partir de la aplicación integra del párrafo segundo del apartado 1, el excedente eventual de recursos propios de las Comunidades con respecto al conjunto de gastos efectivos realizados en el curso de un ejercicio pasará al ejercicio siguiente.

6. La financiación mediante recursos propios de las Comunidades de los gastos relativos a los-programas de investigación de la Comunidad Europea de la Energía Atómica no excluirá la consignación en el presupuesto de las Comunidades de los gastos relativos a los programas complementarios, ni la financiación de dichos gastos por medio de contribuciones financieras de los Estados miembros, determinadas según una clave de reparto especial establecida en virtud de una decisión del Consejo, tomada por unanimidad.

Art. 5. Los ingresos mencionados en el artículo 2, artículo 3, apartados 1 y 2, y artículo 4, apartados 1 a 5 , servirán para financiar indistintamente todos los. gastos consignados en el presupuesto de las Comunidades de conformidad con el artículo 20 del Tratado por el que se constituye un Consejo único y una Comisión única de las Comunidades Europeas.

Art. 6. 1. Los recursos comunitarios a que se refieren los artículos 2, 3 y 4 serán percibidos por los Estados miembros de conformidad con las disposiciones legales, reglamentarias y administrativas nacionales, que serán eventualmente modificadas a tal fin. Los Estados miembros pondrán a disposición de la Comisión dichos recursos.

2. Sin perjuicio del control de cuentas previsto en el articulo 206 del Tratado constitutivo de la Comunidad Económica Europea y de los controles organizados en virtud de la letra c) del artículo 209 de dicho Tratado, el Con- sejo, por unanimidad, a propuesta de la Comisión y previa consulta a la Asamblea, adoptará las disposiciones relativas al control de la recaudación, a la puesta a disposición de la Comisión y a la entrega de los ingresos contemplados en los artículos 2,3 y 4 , así como las modalidades de aplicación del artículo 3 , apartado 3 , y del artículo 4 .

Art. 7. La presente Decisión será notificada a los Estados miembros por el secretario general del Consejo de las Comunidades Europeas y publicada en el Diario Oficial de las Comunidades Europeas.

Los Estados miembros notificarán sin demora al secretario general del Consejo de las Comunidades Europeas el cumplimiento de las formalidades exigidas por sus respectivas normas constitucionales para la adopción de la presente Decisión.

La presente Decisión entrará en vigor el primer día del mes siguiente a la recepción de la última de las notificaciones a que se refiere el párrafo segundo. Sin embargo, si el depósito de los instrumentos de ratificación previstos en el artículo 12 del Tratado por el que se modifican determinadas disposiciones presupuestarias de los Tratados constitutivos de las Comunidades Europeas y del Tratado por el que se constituye un Consejo único y una Comisión única de las Comunidades Europeas no se hubiere efectuado antes de esta fecha por todos los Estados miembros, la presente Decisión entrará en vigor el primer día del mes siguiente al depósito del último de dichos instrumentos de ratificación.

Hecho en Luxemburgo, el 21 de abril de 1970.

Por el Consejo, el Presidente,

P. Harmel. 